\title{
QUEEN'S
UNIVERSITY
BELFAST
}

\section{Synergistic targeting and efficient photodynamic therapy based on graphene oxide quantum dot-upconversion nanocrystal hybrid}

nanoparticles

Liu, Y., Xu, Y., Geng, X., Huo, Y., Chen, D., Sun, K., Zhou, G., Chen, B., \& Tao, K. (2018). Synergistic targeting and efficient photodynamic therapy based on graphene oxide quantum dot-upconversion nanocrystal hybrid nanoparticles. Small, [1800293]. https://doi.org/10.1002/smll.201800293

Published in:

Small

Document Version:

Peer reviewed version

Queen's University Belfast - Research Portal:

Link to publication record in Queen's University Belfast Research Portal

Publisher rights

@ 2018 WILEY-VCH Verlag GmbH \& Co.

This work is made available online in accordance with the publisher's policies. Please refer to any applicable terms of use of the publisher.

\section{General rights}

Copyright for the publications made accessible via the Queen's University Belfast Research Portal is retained by the author(s) and / or other copyright owners and it is a condition of accessing these publications that users recognise and abide by the legal requirements associated with these rights.

\section{Take down policy}

The Research Portal is Queen's institutional repository that provides access to Queen's research output. Every effort has been made to ensure that content in the Research Portal does not infringe any person's rights, or applicable UK laws. If you discover content in the

Research Portal that you believe breaches copyright or violates any law, please contact openaccess@qub.ac.uk. 


\section{WILEY-VCH}

DOI: $10.1002 /$ ((please add manuscript number))

Article type: Full Paper

Synergistic targeting and efficient photodynamic therapy based on graphene oxide quantum dot-upconversion nanocrystal hybrid nanoparticles

Yan Liu†, Yawen Xu†, Xiangshuai Geng†, Yingying Huo, Dexin Chen, Kang Sun, Guangdong Zhou*, Biqiong Chen*, Ke Tao*

Y. Liu, D. Chen, Prof. K. Sun, Dr. K. Tao

State Key Lab of Metal Matrix Composites,

Shanghai Jiao Tong University,

Shanghai 200240,

P. R. China

E-mail:ktao@sjtu.edu.cn

Y. Xu, Y. Huo, Prof. G. Zhou

Department of Plastic and Reconstructive Surgery,

Shanghai $9^{\text {th }}$ People's Hospital,

Shanghai Jiao Tong University School of Medicine,

Shanghai 200235,

P. R. China

E-mail: guangdongzhou@ 126.com

X. Geng

Department of Materials Science and Engineering,

University of Sheffield,

Mappin Street, Sheffield, S1 3JD, United Kingdom.

Prof. B. Chen

School of Mechanical and Aerospace Engineering,

Queen's University Belfast,

Stranmillis Road, Belfast BT9 5AH, United Kingdom.

E-mail: b.chen@qub.ac.uk

Keywords: photodynamic therapy, upconversion nanoparticles, graphene oxide quantum dots, synergistic targeting, subcellular location 


\section{WILEY-VCH}

\section{Abstract}

Locating nanotherapeutics at the active sites, especially in the subcellular scale, is of great importance for nanoparticle-based photodynamic therapy (PDT) and other nanotherapies. However, subcellular targeting agents are generally nonspecific, despite the fact that the accumulation of a nanoformulation at active organelles leads to better therapeutic efficacy. We herein designed a PDT nanoformulation by using graphene oxide quantum dots (GOQDs) with rich functional groups as both the supporter for dual targeting modification and the photosensitizer for generating reactive oxygen species, and upconversion nanoparticles (UCNs) as the transducer of excitation light. A tumor-targeting agent, folic acid, and a mitochondrion-targeting moiety, carboxybutyl triphenylphosphonium, were simultaneously attached onto the UCNs-GOQDs hybrid nanoparticles by surface modification, and a synergistic targeting effect was obtained for these nanoparticles according to both in vitro and in vivo experiments. More significant cell death and a higher extent of mitochondrion damage were observed compared to the results of UCNs-GOQDs nanoparticles with no or just one targeting moiety. Furthermore, the PDT efficacy on tumor-bearing mice was also effectively improved. Overall, the current work presented a synergistic strategy to enhance subcellular targeting and the PDT efficacy for cancer therapy, which may also shed light on other kinds of nanotherapies. 


\section{WILEY-VCH}

\section{Introduction}

Photodynamic therapy (PDT) is a light-triggered strategy for tumor-ablative and functionsparing oncologic intervention, and has been widely used for the treatment of a number of tumors $^{[1]}$, including lung cancer in early stage, ${ }^{[2]}$ Barrett's esophagus, ${ }^{[3]}$ endobronchial tumors, ${ }^{[4]}$ bladder, ${ }^{[5]}$ head and neck, ${ }^{[6]}$ and skin cancers ${ }^{[7]}$. In PDT, photosensitizers (PSs) absorb the energy of light to catalyze surrounding oxygen molecules, so as to produce cytotoxic reactive oxygen species (ROS) and subsequently damage the localized cells. Because neither PSs nor the light employed in PDT has any toxic effects on biological systems, the side-effect on healthy tissues could be avoided by illuminating only tumor lesions. So, unlike conventional cancer treatment methods, PDT has its own merits in minimal invasiveness and repeatability without cumulative toxicity, and it is, therefore, considered as a promising therapeutic modality for cancer ${ }^{[8]}$.

The efficacy of PDT strongly relies on the selective accumulation of PSs at the active sites $^{[9]}$. As an emerging platform for PDT, nanoparticles serving as the carrier can increase the dose of PSs delivered to tumor tissues and facilitate their retention, thus making a significant contribution to enhancing the efficacy of PDT. Konan ${ }^{[10]}$ showed a three-fold higher photocytotoxicity of porphyrin to mammary tumor cells with nanoparticles as its carrier in comparison with the case with no carrier, and similar results were also found for other kinds of $\mathrm{PSs}^{[11]}$. Furthermore, PSs in nanoformulations could be accumulated in tumor tissue in a relatively efficient manner as nanoparticles can be modified with tumor-targeting ligands. With modification of octreotide ${ }^{[12]}$ and $\alpha v \beta_{3}$ integrin-targeting peptide ${ }^{[13]}$, PS-loaded nanoparticles showed a five-fold improvement on tumor accumulation and a $25 \%$ increase of the tumor inhibition ratio compared to untargeted conjugations. Furthermore, the lifespan of ${ }^{1} \mathrm{O}_{2}$ in a biological environment is less than $4 \mu \mathrm{s}^{[14]}$ and the maximal length that ${ }^{1} \mathrm{O}_{2}$ can travel does not exceed $20 \mathrm{~nm}^{[14 \mathrm{~b}-\mathrm{d}, 15]}$, so the effective damage area from PDT therapy is only limited 


\section{WILEY-VCH}

to the subcellular organelles surrounding the tumor tissue. Among these organelles, mitochondria are the power station of life which contribute immensely to cellular mortality management ${ }^{[16]}$, hence they are regarded as a privileged subcellular target. Zhang ${ }^{[17]}$ reported that the cell-killing ratio of the PS-loaded upconversion nanoparticles modified with mitochondrion-targeting peptide had a four-fold increase in contrast to the value for the untargeting nanoformation. Other reports including ours ${ }^{[18]}$ respectively modified different nanoformulations with a mitochondrion-targeting agent, carboxybutyl triphenylphosphonium (TPP), achieved better therapeutic effects compared to untargeting and tissue-scale targeting therapeutics.

However, a noteworthy issue is that the subcellular targeting agents are nonspecific. For example, Zhao ${ }^{[19]}$ demonstrated that mitochondrion-targeting peptide could be largely internalized into various health cell lines such as Caco-2 cells, N2A cells, mouse liver cells, and heart cells of guinea pig. Meanwhile, TPP also enhanced drug penetration and mitochondrion-targeting in RAW 264.7 cells $^{[20]}$, mouse embryonic cells ${ }^{[21]}$, and bovine aortic endothelial cells ${ }^{[22]}$, besides Hela tumor cells ${ }^{[23]}$. These studies strongly suggested that mitochondrion-targeting moieties would non-specifically induce therapeutic drugs into cells, no matter healthy or cancerous, during body circulation, resulting in a loss of the nanoformulation accumulated at the tumor site. Therefore, a mitochondrion-targeting nanoformulation based on active homing to tumor tissue is highly demanded for further elevating the therapeutic efficacy. One possible strategy is to simultaneously integrate tumorspecific ligand, mitochondrion-targeting agent, together with a PS into the nanocarrier, forming a conjugation for synergistic targeting. In this way, the nanoformulations could be actively tailored to target the tumor site by the tumor homing ligand ${ }^{[24]}$, and accumulate near the mitochondria of cancer cells by the subcellular targeting moiety consequently. The key to the success of this strategy is that either the PS agent or the nanocarrier should possess a large 


\section{WILEY-VCH}

number of functional groups so that it can be modified with various kinds of targeting molecules.

Graphene oxide quantum dots (GOQDs), owing to their extraordinary physicochemical, optical properties and biocompatibility, have been widely investigated in biomedical fields such as ultrafast DNA sequencing ${ }^{[25]}$, drug/gene delivery ${ }^{[26]}$, photoactive therapy ${ }^{[27]}$ and bioimaging $^{[25 b, 26 a]}$. More specifically, recent studies found that some kinds of GOQDs are able to generate reactive ${ }^{1} \mathrm{O}_{2}$ upon light illumination ${ }^{[28]}$ with a quantum yield of $\sim 1.3$, which is much higher than classical PSs ${ }^{[29]}$. Based on their large surface area and abundant oxygencontaining functional groups such as hydroxyl, carboxyl and epoxy groups, ${ }^{[30]}$ GOQDs could play as not only an efficient PS but also a supporter for multiple modifications. Meanwhile, upconversion nanoparticles (UCNs) that can be excited by near infrared (NIR) light and emit visible light could serve as a light transducer taking advantage of the deep-penetration of NIR for bio-tissues ${ }^{[31]}$. Therefore, we conjugated GOQDs and UCNs together in our nanoformation design, and simultaneously attach a typical tumor-targeting ligand, folic acid (FA), and a mitochondrion-targeting agent, TPP, with the help of the sufficient functional groups on GOQDs, as shown in Figure 1. We found this nanoformulation accumulated at the active sites more effectively compared with their individually targeting counterparts, which indicated the synergistic effect of multiple targeting strategy resulted in an improved therapeutic efficacy.

\section{Results and Discussion}

\subsection{Synthesis and characterization}

Graphene oxide quantum dots were synthesized by a modified strong-acid oxidation $\operatorname{method}^{[32]}$. The size of the resultant GOQDs was approximately $30 \mathrm{~nm}$ and the thickness was between 2 and 3 nm (Figure S1a, b and c). From Fourier transform infrared (FTIR) spectrum 


\section{WILEY-VCH}

(Figure S1d), abundant oxygen-containing groups including carbonyl and carboxyl $(\mathrm{C}=\mathrm{O}$ stretching vibration: $\left.1720 \mathrm{~cm}^{-1}\right)$, hydroxyl $\left(3442 \mathrm{~cm}^{-1}\right)$, and epoxy groups $\left(1255 \mathrm{~cm}^{-1}, 1114\right.$ $\left.\mathrm{cm}^{-1}\right)$ can be verified. The production of singlet oxygen $\left({ }^{1} \mathrm{O}_{2}\right)$, as the dominant part of ROS, was measured by 9,10-anthracenediyl-bis(methylene)dimalonic acid (ABDA) as an indicator, because the UV-Vis absorption of ABDA will decay with the generation of ${ }^{1} \mathrm{O}_{2}{ }^{[33]}$. In the measurement, Rose Bengal (RB) was taken as the reference photosensitiser ${ }^{[29,34]}$. As shown in Figure S1e, the attenuation of ABDA absorption in GOQD solution was $\sim 3$ times faster than RB solution. To quantify the ${ }^{1} \mathrm{O}_{2}$ quantum yield of GOQDs, the phosphorescence of the generated ${ }^{1} \mathrm{O}_{2}$ at $1280 \mathrm{~nm}^{[29,35]}$ was measured in deuterium oxide $\left(\mathrm{D}_{2} \mathrm{O}\right)$ under 546-nm irradiation, and the result showed that GOQDs have a much stronger NIR emission than RB (Figure S1f), indicating a higher ${ }^{1} \mathrm{O}_{2}$ production. According to the integration of fluorescence intensity, the quantum yield of GOQDs was calculated to be $\sim 1.56$, which is slightly higher than the reported result $(\sim 1.3)^{[29]}$ and more than twice higher than that of $\mathrm{RB}\left(0.76^{[36]}\right)$. Compared to the reported graphene quantum dots synthesized by hydrothermal method ${ }^{[29]}$, GOQDs synthesized in current work contain abundant oxygenated functional groups which might help the oxygen quenching of triplet states so as to increase the ${ }^{1} \mathrm{O}_{2}$ quantum yield from multistate sensitization $^{[29]}$ of GOQDs.

$\mathrm{NaYF}_{4}: \mathrm{Yb}, \mathrm{Tm} @ \mathrm{NaGdF}_{4}$ nanocrystals as the UCNs were synthesized by a successive hot injection method following our previous work $^{[37]}$, and were modified by (3-aminopropyl) triethoxysilane (APTES) ${ }^{[38]}$ in order to obtain $-\mathrm{NH}_{2}$ functional groups on the surface for later conjugation with GOQDs. TEM images of the as-prepared UCN crystals were shown in Figure S2. The average diameter of the hexagonal nanoparticles was $\sim 50 \mathrm{~nm}$. The distribution of yttrium (Y) well matched the inner boundary of gadolinium (Gd) in the energy dispersive spectroscopy (EDS) elemental mapping (Figure 2a), indicating a core-shell 


\section{WILEY-VCH}

structure. Meanwhile, the successful coating of silica layer on UCNs can be confirmed by the distribution of Si just outside Gd (Figure 2a).

The UCNs with amine groups were then conjugated with GOQDs via the EDC-NHS activating method, and the successful conjugation was evidenced by the emerging peaks on FTIR absorption spectrum at $1714 \mathrm{~cm}^{-1}(\mathrm{C}=\mathrm{O}), 1511 \mathrm{~cm}^{-1}(\mathrm{~N}-\mathrm{H})$ and $1454 \mathrm{~cm}^{-1}(\mathrm{C}-\mathrm{N})$ compared to UCNs (Figure S3a). Meanwhile, the new UV-Vis absorption peak of UCNsGOQDs nanohybrid at $220 \mathrm{~nm}$ further proved successful conjugation owing to the characteristic peak of around 216-220 nm of GOQDs (Figure S3b). The payload of GOQDs in UCNs-GOQDs conjugation was measured by thermogravimetric analysis (TGA) (Figure S4). Since the weight of UCNs-GOQDs nanohybrid maintained almost stable after $500{ }^{\circ} \mathrm{C}$, we roughly estimated the weight percentage of GOQDs to UCNs to be $\sim 1.4 \mathrm{wt} \%$ by comparing the weight loss of UCNs-GOQDs to the weight losses of GOQDs and UCNs at $500{ }^{\circ} \mathrm{C}$.

FA and TPP were further attached onto the surface of UCNs-GOQDs nanohybrid in succession to form a doubly targeting nanohybrid (denoted as UCNs-GQODs-FA/TPP), both of which were conducted through linking EDC activated carboxyl groups of TPP or FA to the functional groups on GOQDs. For comparison, FA and TPP were also individually used for modifying UCNs-GOQDs by the same protocol to obtain two control groups (denoted as UCNs-GOQDs-FA and UCNs-GOQDs-TPP, respectively). For the samples consisting of FA, the successful modification can be confirmed by the UV-vis absorption peak around $280 \mathrm{~nm}$, as the absorption peak of FA is around $288 \mathrm{~nm}$ (Figure $2 \mathrm{~b}$ and Figure S3c). Meanwhile for the samples containing TPP, the integration can also be confirmed by the absorption peak at $\sim 263$ $\mathrm{nm}$ (Figure $2 \mathrm{~b}$ and Figure S3d). Furthermore, the distribution of phosphorus element in UCNs-GOQDs-FA/TPP hybrid nanoparticles by EDS element mapping (Figure 2a) also proved the successful linking of TPP. 


\section{WILEY-VCH}

We further characterized the nanohybrids by dynamic light scattering (DLS). As shown in Figure S3f, the average hydrodynamic size of APTES-modified UCNs is about $83.2 \mathrm{~nm}$, and the zeta potential was about $+23 \mathrm{mV}$. Meanwhile, the average hydrodynamic sizes of UCNsGOQDs, UCNs-GOQDs-FA, UCNs-GOQDs-TPP and UCNs-GOQDs-FA/TPP are about 109 $\mathrm{nm}, 126 \mathrm{~nm}, 121 \mathrm{~nm}$ and $131 \mathrm{~nm}$, respectively, all of which are larger than UCNs owing to the successful conjugation with GOQDs and the surface modification. As the hydrodynamic size (corresponding to the UCNs and the swollen corona of the surface coating) is slightly larger than the size from TEM images (only corresponding to UCNs), it should be concluded that the nanohybrids were well dispersed in water. The stability of the nanohybrid suspension was further observed by taking photos of the suspensions with a $\mathrm{Gd}^{3+}$ concentration of 300 ppm (or $\sim 0.84 \mathrm{mg} / \mathrm{ml}$ nanohybrid) in deionized water and DMEM after $24 \mathrm{~h}$. All the suspensions did not present any obvious precipitate, as shown in Figure S5, indicating their stability in both water and cell culture medium.

The ${ }^{1} \mathrm{O}_{2}$ production of UCNs-GOQDs-FA/TPP, as well as UCNs-GOQDs, UCNs-GOQDsFA and UCNs-GOQDs-TPP, was measured by the above method with ABDA as an indicator and the results were shown in Figure 3a. After irradiated by $980 \mathrm{~nm}\left(1 \mathrm{~W} / \mathrm{cm}^{2}\right)$ for $10 \mathrm{~min}$, the absorption intensity of ABDA for all these four samples decreased to $\sim 72 \%$, indicating that the modification has almost no influence on the generation of ${ }^{1} \mathrm{O}_{2}$. Due to the lack of a standard quantification method for the ROS generation of UCN-loaded photosensitizers, it is hard to compare the current work with others' works. Nevertheless, the ROS generation in this work is much higher than our previous work under all the same conditions, in which the absorption of ABDA decreased to $\sim 80 \%$ in $10 \min ^{[18]}$. On one hand, the higher ${ }^{1} \mathrm{O}_{2}$ quantum yield of GOQDs could lead to the higher amount of ROS under certain illumination than classical photosensitizers. On the other hand, the absorption of GOQDs covers a wide range of wavelengths from $\sim 200 \mathrm{~nm}$ to $\sim 500 \mathrm{~nm}$, indicating that the energy of two main emission 


\section{WILEY-VCH}

bands of UCNs (330 370 nm, 440 490 nm) could be absorbed by GOQDs. To confirm this postulation, the fluorescence of the nanohybrids was measured to make a comparison with that of unconjugated UCNs. After conjugating with GOQDs, the intensity of the emission peaks at $365 \mathrm{~nm}$ and $488 \mathrm{~nm}$ decreased to $\sim 50 \%$ (Figure $3 \mathrm{~b}$ ), while the emission peak at $\sim 800$ nm was kept almost the same. Furthermore, the emission was almost the same for UCNsGOQDs-FA, UCNs-GOQDs-TPP and UCNs-GOQDs-FA/TPP hybrids, indicating that the modification with FA and TPP has no obvious effect on the energy transfer efficiency.

\subsection{In vitro targeting and PDT}

The synergistic targeting effects were evaluated in vitro in terms of cellular uptake (Figure 4a). After incubation with Hela cells for 4 hours, the UCNs-GOQDs nanohybrids inside cells were about $2.08 \mu \mathrm{g}$ UCNs per $10^{4}$ cells. For the conjugations individually modified with FA or TPP, the cellular uptake increased to $\sim 3.28 \mu \mathrm{g} / 10^{4}$ cells (an increase of $\sim 58 \%$ ) and $\sim 2.82$ $\mu \mathrm{g} / 10^{4}$ cells (an increase of $\sim 36 \%$ ), respectively. With multiple targeting moieties, the cellular uptake further increased to about $3.95 \mu \mathrm{g} / 10^{4}$ cells (an increase of $\sim 90 \%$ as compared to UCNs-GOQDs), indicating that the combination of different targeting agents significantly enhances the uptake efficacy in Hela cells. For comparison, we first incubated the Hela cells with folic acid and measure the cellular uptake with inhibiting the folate receptor on cell membrane. It was found that the endocytosis for UCNs-GOQDs and UCNs-GOQDs-TPP presented almost no obvious difference between the cells with and without folate receptor inhibition. However, for UCNs-GOQDs-FA, the cellular uptake decreased from $\sim 3.3 \mu \mathrm{g} / 10^{4}$ cells (without the inhibition) to $\sim 1.9 \mu \mathrm{g} / 10^{4}$ cells (with the inhibition). For UCNs-GOQDsFA/TPP, the cellular uptake also decreased $\sim 25 \%$ after inhibiting folate receptor, which was close to the uptake of UCNs-GOQDs-TPP and still higher than that of UCNs-GOQDs. These results indicated that the modification of FA facilitates the internalization of nanohybrids via folate receptor-mediated endocytosis. Furthermore, human fibroblast cells were also chosen 


\section{WILEY-VCH}

as a control group, and the cellular uptake was determined as $2.41 \mu \mathrm{g}, 2.55 \mu \mathrm{g}, 3.31 \mu \mathrm{g}$ and $3.47 \mu \mathrm{g}$ UCNs per $10^{4}$ cells for UCNs-GOQDs, UCNs-GOQDs-FA, UCNs-GOQDs-TPP, UCNs-GOQDs-FA/TPP, respectively. For fibroblast cells, the increase of cellular uptake with FA modified UCNs-GOQDs was almost negligible, indicating FA is specific for folate receptor-overexpressed cancer cells. In contrast, it should be noted that TPP modification resulted in a $\sim 37 \%$ increase as compared to that of UCNs-GOQDs, which is similar to that of Hela cells and demonstrates the nonspecific feature of TPP modification.

The Hela cells without inhibiting folate receptor were also observed by TEM (Figure 4b) after incubation with different samples. Without targeting moiety, few UCNs-GOQDs nanohybrids were found in cytoplasm. When modified with FA molecules, the nanohybrids were endocytosed into cells with an increased amount. In particular, when modified with TPP, there is a tendency for these nanoparticles to accumulate near mitochondria. With both FA and TPP modifications, the amount of endocytosis to cytoplasm and mitochondria was further improved, indicating the high synergistic targeting efficiency of UCNs-GOQDs-FA/TPP.

The synergistic targeting and localization of the nanohybrids were further observed from confocal fluorescence images of Hela cells stained with mitochondrial dye JC-1 ${ }^{[39]}$ (Figure 4c and Figure S6). JC-1 displays potential-dependent accumulation in the normal mitochondria accompanied by the red fluorescence emission due to the formation of JC-aggregates ${ }^{[14 b]}$. Therefore, the mitochondrion targeting efficiency could be assessed by the colocalization of the red fluorescence from aggregated JC-1 to that from UCNs. As shown in Figure 4c, the percentages of mitochondrial colocalization of UCNs-GOQDs-FA and UCNs-GOQDs-TPP were $35.1 \%$ and $34.0 \%$, respectively, both of which were much higher than the value of UCNs-GOQDs (18.4\%). Meanwhile, in UCNs-GOQDs-FA/TPP group the colocalization increased to $51.0 \%$, which is $\sim 2.8$ times higher than UCNs-GOQDs and $\sim 1.5$ times higher 


\section{WILEY-VCH}

than the monomeric targeted groups, indicating more nanohybrids were delivered to the sites around mitochondria by the synergistic targeting.

FA has been widely acknowledged for the enhancement of receptor-mediated endocytosis of FR-overexpressed tumor cells ${ }^{[40]}$. Meanwhile, the homing of cationic TPP moiety modified nanoparticles into cytoplasm was considered to be driven by electrostatic interaction, as the membrane potential of mitochondria is highly negative $(-140 \mathrm{mV} \text { to }-180 \mathrm{mV})^{[41]}$. Therefore, the modification of nanoparticles with FA showed an enhanced cellular uptake for Hela cells without receptor inhibition, whilst TPP modification led to an increase of internalization for both Hela cells (whether inhibited or not) and fibroblast cells. In addition, in the endocytosis for UCNs-GOQDs-FA, the nanoparticles were internalized through receptor-dependent caveolae-mediated endocytosis $^{[42]}$, in which the pathway does not involve the lysosomes and the vesicular cargo would be delivered through cytoplasm into other subcellular compartments $^{[43]}$. As a result, UCNs-GOQDs-FA/TPP could escape from lysosomes and accumulated near mitochondria with a higher amount ${ }^{[44]}$. Besides, the enhancement in cellular internalization and mitochondria targeting from TPP modification, following another mechanism (electrostatic interaction), may still remain, and combining these two different pathways, both cellular uptake and mitochondria targeting efficiency would be significantly enhanced.

To investigate NIR triggered PDT effect of UCNs-GOQDs-FA/TPP hybrid nanoparticles, Hela cells were irradiated by $980 \mathrm{~nm}$ laser $\left(1 \mathrm{~W} / \mathrm{cm}^{2}\right)$ for $15 \mathrm{~min}$ after the uptake of nanohybrids. As shown in Figure 5a and Figure S7, without the irradiation of NIR, the cell viability slightly decreased to $\sim 80 \%$ when the concentration of $\mathrm{Gd}^{3+}$ increased to $420 \mathrm{ppm}(\sim 2$ $\mathrm{mg} / \mathrm{mL}$ of UCNs). After irradiated (Figure $5 \mathrm{~b}$ ) for $15 \mathrm{~min}$, the cell viability for all the modified groups decreased faster than the unmodified group of UCNs-GOQDs. Meanwhile, the viability of the cells incubated with UCNs-GOQDs-TPP is lower than that with UCNs- 


\section{WILEY-VCH}

GOQDs-FA group under the same conditions, indicating UCNs-GOQDs-TPP that targets the mitochondria sites may possess better therapeutic effect than UCNs-GOQDs-FA with similar cellular internalization, which is coincident with our previous report ${ }^{[18]}$. Furthermore, among all groups, the decrease of cell viability for UCNs-GOQDs-FA/TPP group is the most significant, which is attributed to the enhanced uptaken dose and mitochondrial localization.

To further investigate the capability of killing cancer cells under illumination, flow cytometric analysis of the Annexin V-FITC/PI labeling assay ${ }^{[45]}$ was conducted. As shown in Figure 5c, the percentage of cells at each corresponding zone was marked. Without irradiation, the live Hela cells in four samples were all higher than $80 \%$. After irradiated for 10 min, live cells were decreased to $72.6 \%$ in UCNs-GOQDs group. Meanwhile, the percentages of live cells were $60.8 \%$ and $58.0 \%$ in UCNs-GOQDs-FA and UCNs-GOQDsTPP groups. In UCNs-GOQDs-FA/TPP group, the percentage of remained live cells was only about 37.4\%. In TPP modification group, the apoptosis is more serious than in FA modification group, indicating a higher sensitive response of mitochondria to PDT process. Meanwhile the synergistic targeting showed the highest percentage of cell death, which was consistent with the result of cell viability of in-vitro PDT and further proved the efficient therapeutic effect induced by synergistic targeting.

Since JC-1 forms red emissive fluorescent aggregates in normal mitochondria with a high membrane potential (MMP) and change to green emissive monomer in damaged mitochondria with a low $\mathrm{MMP}^{[46]}$, the change of fluorescence intensity of aggregate JC-1 and monomeric JC-1 could be utilized to evaluate the health state of mitochondria (Figure 4c and Figure S8). The increase of green fluorescence of monomeric JC-1 in UCNs-GOQDs-TPP group is more significant than that of UCNs-GOQDs-FA group, suggesting more mitochondrial apoptosis of the former group than the latter one. Meanwhile, both FA and TPP modified groups showed more obvious mitochondrial damage than the UCNs-GOQDs group. In UCNs-GOQDs- 


\section{WILEY-VCH}

FA/TPP group, the green fluorescence of monomeric JC-1 was further increased. These results gave a proof of the improved damage of mitochondria in the occasions with TPP modification and especially, synergistic targeting.

\subsection{Synergistic targeting and PDT in vivo}

In the in vivo experiments, UCNs mediated conjugations were injected intraperitoneally into mice with bearing tumors at an injection dose (ID) of $18 \mathrm{mg} / \mathrm{kg}$. The doses of nanoparticles accumulating at the tumor lesion were measured as $0.09 \%$ ID/g for UCNs-GQODs, $0.15 \%$ ID/g for UCNs-GQODs-FA (increased for $~ 73 \%$ ), $0.12 \%$ ID/g for UCNs-GQODs-TPP (increased for $\sim 35 \%$ ), and $0.21 \%$ ID/g for UCNs-GQODs-FA/TPP (increased for $\sim 145 \%$ ), respectively (Figure 6a). These results on one hand further suggested that only modification with mitrochondrion-targeting ligand (TPP) resulted in a lower accumulation of nanoparticle in tumor lesion, and on the other hand indicated the positive outcome of the targeting effect by dual targeting moieties. Meanwhile, to determine whether the nanoparticles were internalized into cells, the extracellular matrix of the tumor tissue with intratumoral injection administration was digested by $0.15 \%$ collagenase and resultant cells were purified, resuspended in PBS, and stained by rhodamine 123 for mitochondria and Hoechst 33258 for nuclei. As shown in Figure 6b, the fluorescence of UCNs was observed just around the cell nuclei and to some extent overlapped with that of rhodamine 123 , indicating part of the nanoparticles were uptaken by the cells. By using software imageJ, the colocalization coefficients of rhodamine 123 and UCNs were analyzed, and the results were $0.33,0.38,0.40$, 0.44, for UCNs-GQODs, UCNs-GQODs-FA, UCNs-GQODs-TPP, UCNs-GQODs-FA/TPP, respectively. It should be noted that despite the targeted amount of UCNs-GQODs-TPP to tumor lesion was lower than that of UCNs-GQODs-FA, the colocalization coefficient of the former was still comparable to or slightly higher than the latter, indicating the mitochondria targeting effect of TPP modification in vivo. Moreover, the dual modification with FA and 


\section{WILEY-VCH}

TPP leading to the synergistic targeting could be further evidenced and more nanoparticles would be in a close proximity to mitochondria.

Having verified the advantage of dually modified platform in tissue targeting, in vivo therapeutic efficiency was studied. The tumors were irradiated by $980 \mathrm{~nm}$ laser $\left(0.5 \mathrm{~W} / \mathrm{cm}^{2}\right)$ for $30 \mathrm{~min}$ at $3 \mathrm{~h}$ after the intraperitoneal injection. In the control groups injected with PBS with or without laser, the tumors continuously grew up to near three-fold volume after 15 days (Figure 7), showing negligible effect of laser irradiation. In all the groups without irradiation, the growth of tumor was similar to that of the PBS groups. In UCNs-GOQDs with laser groups, after irradiation by $980 \mathrm{~nm}$ laser for 30min, the tumor still kept on growing while slower than that of PBS group. In the groups with the injection of UCNs-GOQDs-FA and UCNs-GOQDs-TPP, the volume of tumors only slightly increased. In contrast, in the synergistic targeting group, tumors were regressed and the remnant volume of tumor was only about $25 \%$ in 15 days, demonstrating the enhanced PDT efficacy. The therapeutic effect of the synergistic targeting group was further proved by the histological analysis of tumor tissues. Figure 8 showed the tumor slices stained by haematoxylin and eosin at different time points, clearly showing that the tumor cells are diminished, spheroidized and isolated gradually. In the 15-day observation after therapy, the weight of mice was continuously recorded, and no obvious difference was detected among all groups (Figure S9a-b). After 15 days, all the mice were survived. Then the mice were sacrificed and the histological analysis of main organs (heart, liver, spleen, lung, kidney) in UCNs-GOQDs-FA/TPP group (Figure S9d) was conduct, showing the similar results as compared to the control group. The biodistribution of UCNs-GOQDs-FA/TPP was identified after intraperitoneal injection for $3 \mathrm{~h}$, 15 days and 30 days, respectively, as shown in Figure S9c. At 3 hours after the injection, it was found that compared with targeting $0.21 \%$ of the injection dose to tumor lesion, a much higher amount of nanohybrids were delivered to kidney, liver, spleen and lung. It should be 


\section{WILEY-VCH}

noted that after 15 or 30 days, the nanoparticles in these organs were metabolized to a low level, which is comparable to the dose remained in the tumor. Combining with the observation of histological slices, this result indicates negligible side effects of the nanohybrids on main organs.

\section{Conclusions}

With better capability of generating ROS than classical photosensitizers, we loaded GOQDs on UCNs to formed a NIR excited PDT nanoformulation. Meanwhile, owing to the abundant functional groups on GOQDs, FA and TPP, as the tumor targeting and mitochondrial targeting moieties respectively, were simultaneously grafted onto UCNs-GOQDs conjugation. This dual modification led to a higher targeting of nanoparticles to cancer cells, and to tumor lesions in vivo as well. Moreover, the accumulation of nanoparticles near mitochondria for both in vitro and in vivo cases were also observed. With such an enhanced targeting effect, the cell death originated from mitochondria damage as well as the PDT efficacy in vivo were improved. Owing to that targeting efficiency is a general issue not only for PDT but also for other nanotherapeutics, our work, indicating that dual modification based on different targeting pathways may benefit for the accumulation of nanoformulations at the active sites, may be meaningful for the design of nanomedicines. 


\section{WILEY-VCH}

Figure 1. Schematic illustration of the design of the nanohybrids based on UCNs-GOQDs conjugation.

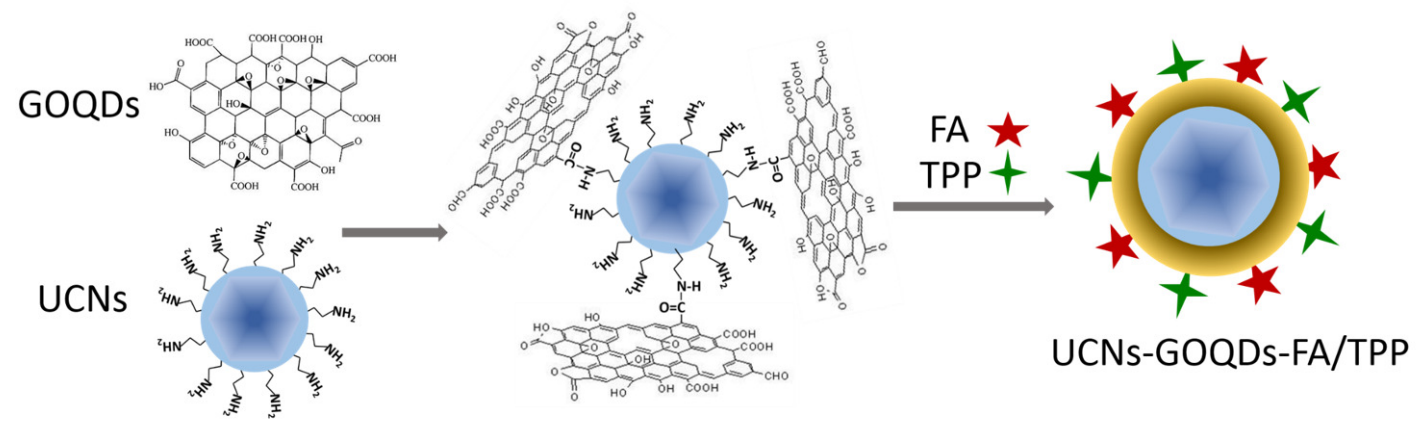




\section{WILEY-VCH}

Figure 2. a) Energy dispersive spectrometer mapping of elements, and b) the UV-Vis absorption of UCNs-GOQDs-FA/TPP.
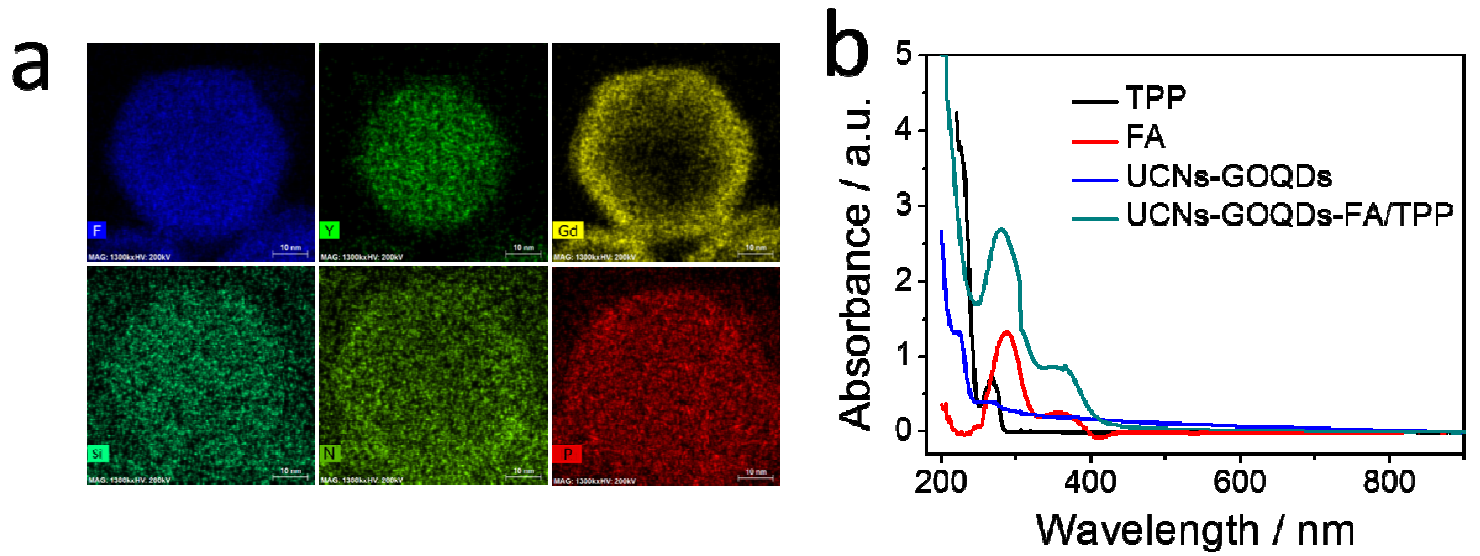


\section{WILEY-VCH}

Figure 3. a) ROS generation of UCNs-GOQDs-FA/TPP detected by ABDA; b) UV-Vis absorption of GOQDs (black) and florescence spectra of UCNs, UCNS-GOQDSs, UCNsGOQDs-FA/TPP, respectively.
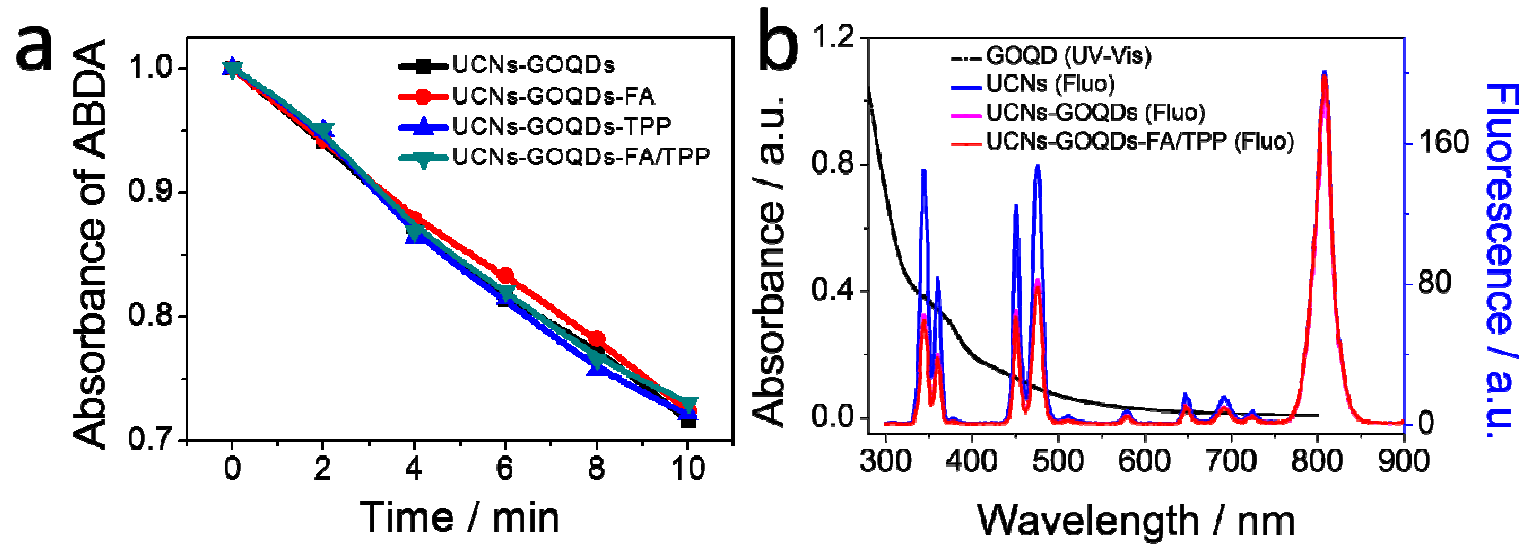


\section{WILEY-VCH}

Figure 4. a) Cellular uptake in Hela cells without (black) and with (blue) FA receptor inhibiting, and human fibroblast cells (red). Data are presented as mean $\pm \mathrm{SD}(\mathrm{n}=3) . * \mathrm{P}<0.05$, $* * \mathrm{P}<0.01$. b) TEM of Hela cells incubated with the nanohybrids. The mitochondria are marked by red arrows and the boundary of nucleus is presented by dashed line. c) Laser scanning confocal microscope (LSCM) images of Hela cells stained by JC-1 after incubation with nanohybrids. Left panel: direct observation without PDT laser irradiation, and right panel: with laser irradiation for $3 \mathrm{~min}$ (Scale bar=50 $\mu \mathrm{m}$ ).

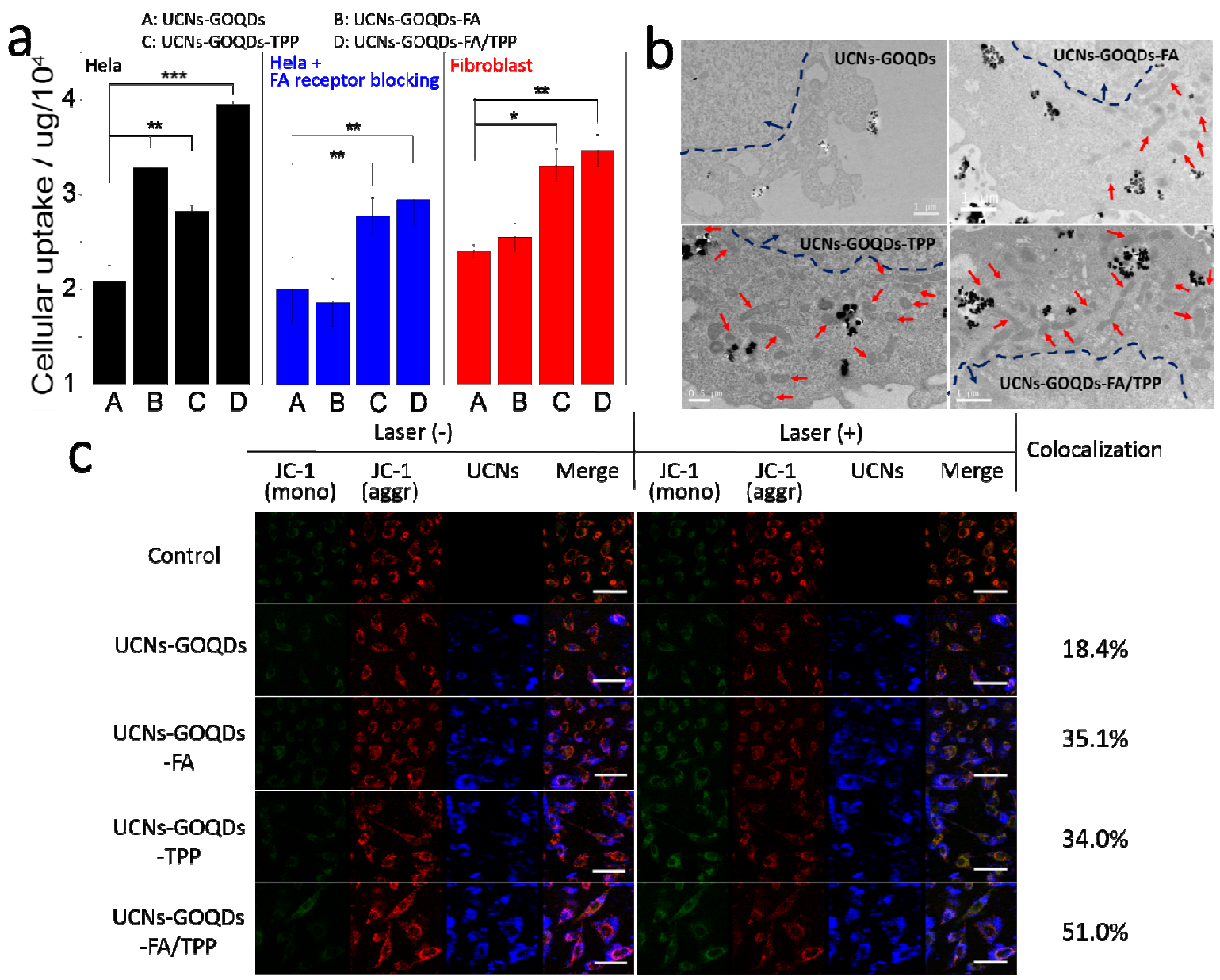




\section{WILEY-VCH}

Figure 5. Cell viability of Hela cells incubated with nanohybrids a) without and b) with 980 nm laser; c) Annexin V-FITC/PI assay of Hela cells by flow cytometry.
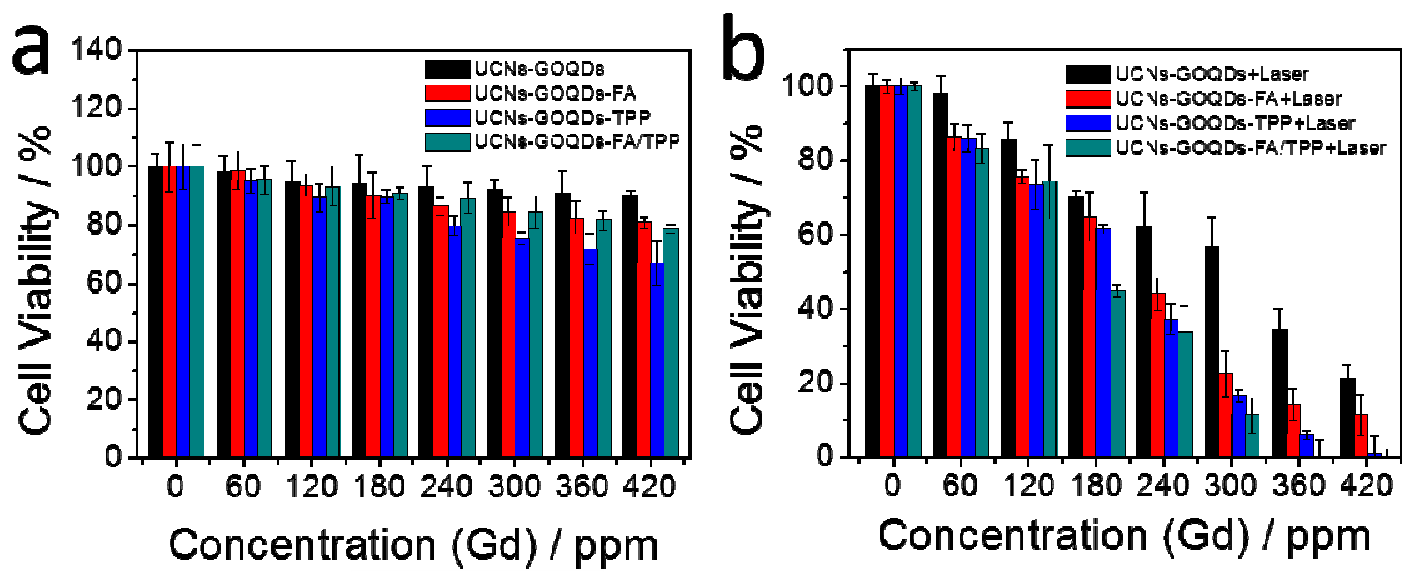

$C$

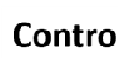

UCNs-GOQDs UCNs-GOQDs

UCNs-GOQDS UCNs-GOQDS

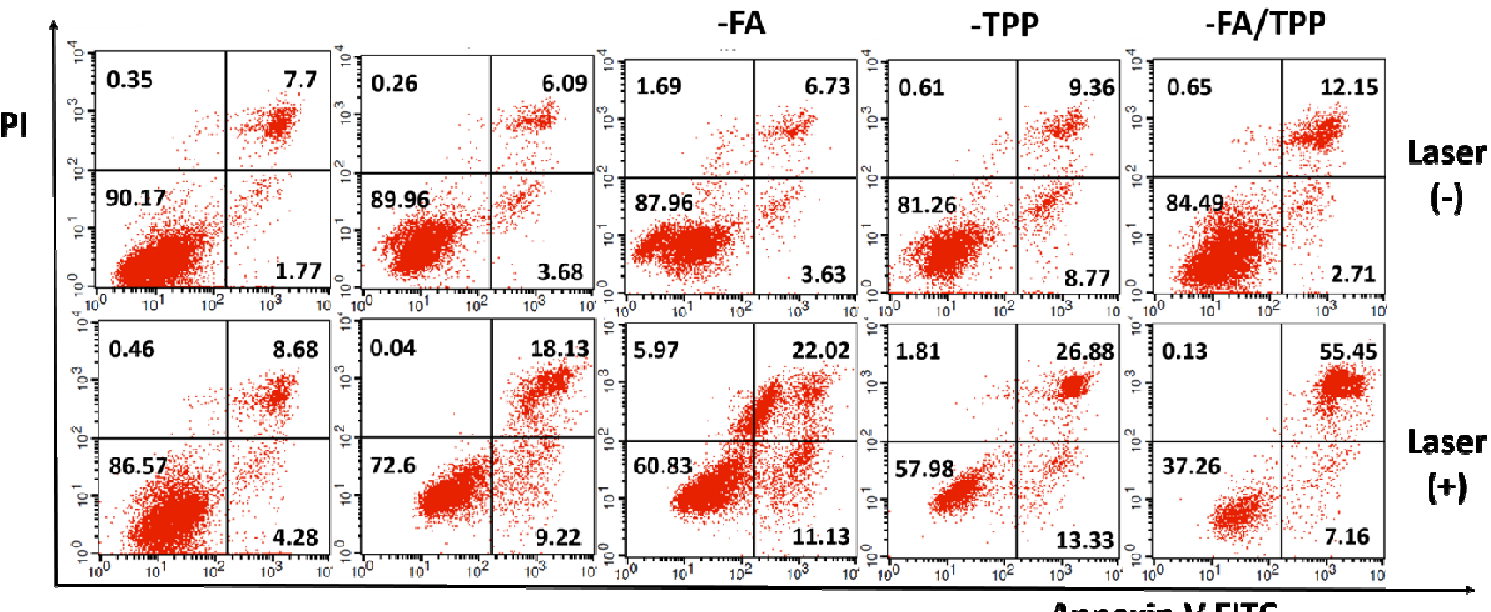

Annexin V FITC 


\section{WILEY-VCH}

Figure 6. Targeted dose of nanohybrid in tumor tissues with intraperitoneal injection. Data are presented as mean $\pm \mathrm{SD}(\mathrm{n}=3)$. $* \mathrm{P}<0.05, * * \mathrm{P}<0.01, * * * \mathrm{P}<0.001$. b) LSCM images of tumor tissue cells suspension. The tumor tissues were removed after intratumoral injection for $3 \mathrm{~h}$, digested by $0.15 \%$ collagenase overnight and stained by Rhodamine 123 and Hoechst 33258 (Scale bar $=20 \mu \mathrm{m})$.
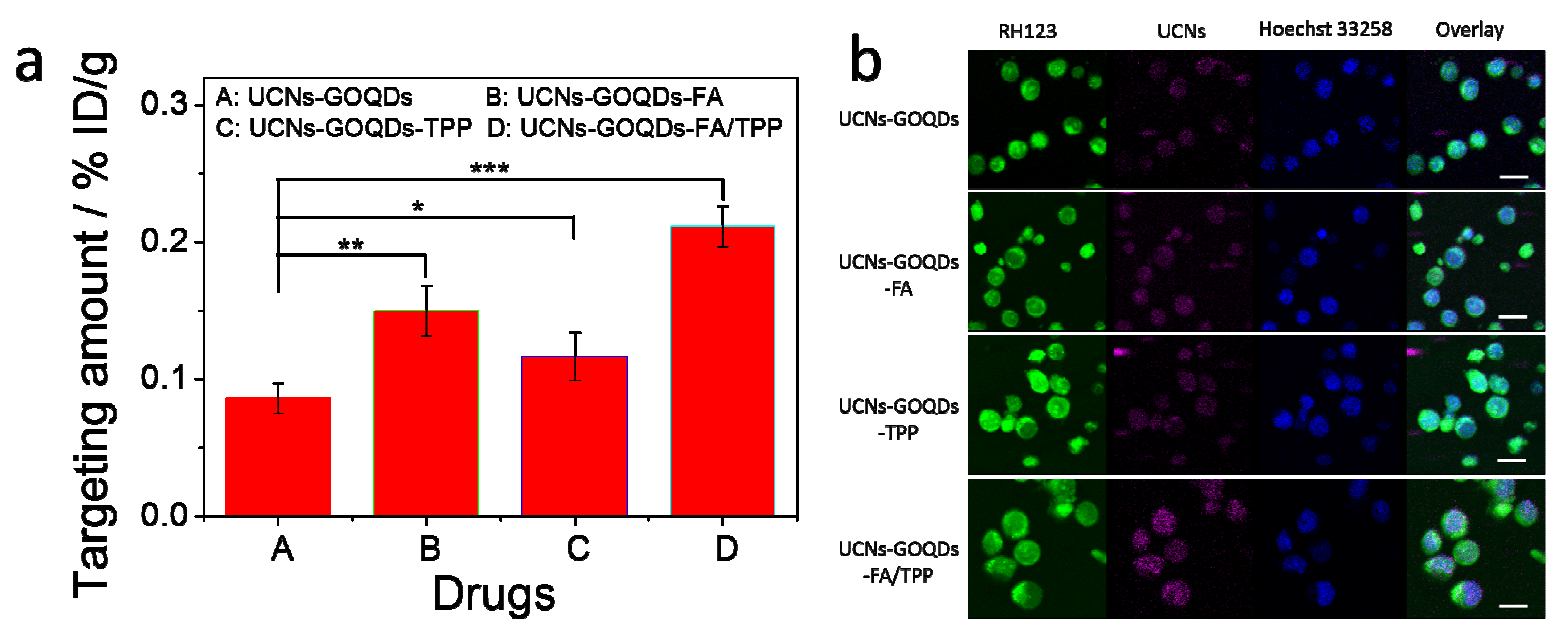


\section{WILEY-VCH}

Figure 7. a) The normalized tumor volume and b) photos of naked mice with different treatments. Data are presented as mean $\pm \mathrm{SD}(\mathrm{n}=6) . * \mathrm{P}<0.05, * * \mathrm{P}<0.01, * * * \mathrm{P}<0.001$.
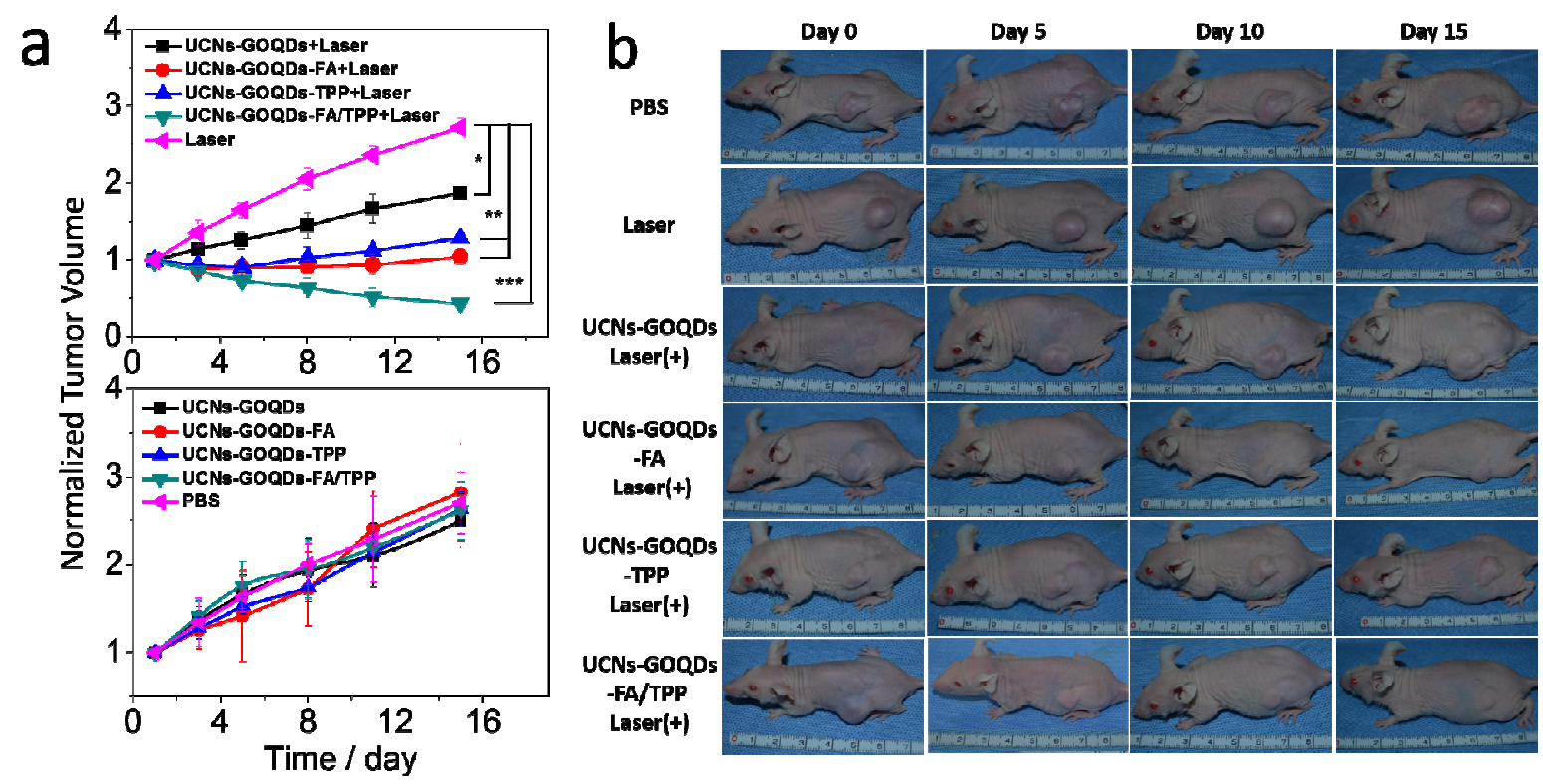


\section{WILEY-VCH}

Figure 8. Histological analysis of hematoxylin-eosin stained tumor sections in UCNsGOQDs-FA/TPP treatment group.

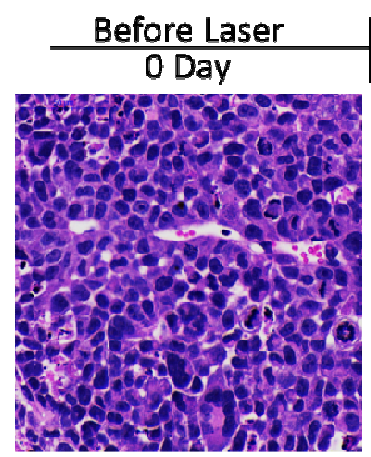

\section{After Laser}

5 Day

10 Day

15 Day

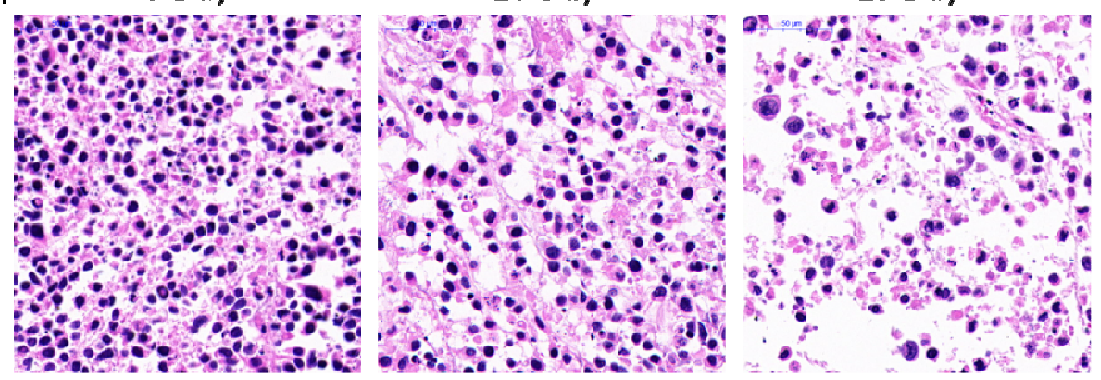




\section{WILEY-VCH}

\section{Supporting Information}

Experimental details, microstructure and physiochemical properties of GOQDs, UCNs, and different nanohybrids respectively, the change of fluorescence of JC-1, and biocompatibility of the nanohybrids.

Supporting Information is available from the Wiley Online Library or from the author.

\section{Acknowledgements}

Y. L., Y. X. and X. G. contributed equally to this work. This work was financially supported by National Science Foundation of China (Project No. 31470958, No. 31671027, and No. 31671004), and a Global Innovation Initiative project (GII207) funded by the British Council and the Department of Business, Innovation and Skills in the United Kingdom. We thank Instrumental Analysis Center of SJTU for assistance with the instrumentation.

Received: ((will be filled in by the editorial staff))

Revised: ((will be filled in by the editorial staff))

Published online: ((will be filled in by the editorial staff)) 


\section{WILEY-VCH}

References

[1] a) J. S. McCaughan, Drugs Aging 1999, 15, 49; b) J. J. Schuitmaker, P. Baas, H. L. L. M. van Leengoed, F. W. van der Meulen, W. M. Star, N. van Zandwijk, J. Photochem.

Photobiol., B 1996, 34, 3.

[2] H. Kato, J. Photochem. Photobiol., B 1998, 42, 96.

[3] a) C. Hur, N. S. Nishioka, G. S. Gazelle, Dig. Dis. Sci. 2003, 48, 1273; b) J. S.

McCaughan Jr, Photomed. Laser Surg. 1996, 14, 223.

[4] J. S. McCaughan, T. E. Williams, B. H. Bethel, Lasers Surg. Med. 1986, 6, 336.

[5] R. J. Skyrme, A. J. French, S. N. Datta, R. Allman, M. D. Mason, P. N. Matthews, BJU Int. 2005, 95, 1206.

[6] D. E. Schuller, J. S. McCaughan, Jr, R. P. Rock, Arch. Otolaryngol. 1985, 111, 351.

[7] L. E. Rhodes, M. de Rie, Y. Enström, R. Groves, T. Morken, V. Goulden, G. A. E.

Wong, J. J. Grob, S. Varma, P. Wolf, Arch. Dermatol. 2004, 140, 17.

[8] S. S. Lucky, K. C. Soo, Y. Zhang, Chem. Rev. 2015, 115, 1990.

[9] S. H. Yun, S. J. J. Kwok, Nature Biomedical Engineering. 2017, 1, 0008.

[10] Y. N. Konan, J. Chevallier, R. Gurny, E. Allémann, Photochem. Photobiol. 2003, 77,

638.

[11] Y. N. Konan-Kouakou, R. Boch, R. Gurny, E. Allemann, J. Controlled Release 2005, $103,83$.

[12] S. He, Z. Zhou, L. Li, Q. Yang, Y. Yang, S. Guan, J. Zhang, X. Zhu, Y. Jin, Y. Huang, Drug Delivery 2016, 23, 285.

[13] A. Ray, N. Larson, D. B. Pike, M. Gruner, S. Naik, H. Bauer, A. Malugin, K. Greish, H. Ghandehari, Mol. Pharmacol. 2011, 8, 1090.

[14] a) G. Palumbo, Expert Opin. Drug Delivery 2007, 4, 131; b) G. Feng, W. Qin, Q. Hu, B. Z. Tang, B. Liu, Adv. Healthcare Mater. 2015, 4, 2667; c) J. Moan, Photochem. Photobiol. 1991, 53, 549; d) P. R. Ogilby, Chem. Soc. Rev. 2010, 39, 3181.

[15] S. Kim, T. Tachikawa, M. Fujitsuka, T. Majima, J. Am. Chem. Soc. 2014, 136, 11707.

[16] J. R. Friedman, J. Nunnari, Nature 2014, 505, 335.

[17] X. Zhang, F. Ai, T. Sun, F. Wang, G. Zhu, Inorg. Chem. 2016, 55, 3872.

[18] D. Chen, R. Tao, K. Tao, B. Chen, S. K. Choi, Q. Tian, Y. Xu, G. Zhou, K. Sun, Small 2017, 13, 1602053.

[19] K. Zhao, G. M. Zhao, D. Wu, Y. Soong, A. V. Birk, P. W. Schiller, H. H. Szeto, J. Biol. Chem. 2004, 279, 34682.

[20] S. Marrache, S. Dhar, Proc. Natl. Acad. Sci. U. S. A. 2013, 110, 9445.

[21] V. E. Kagan, P. Wipf, D. Stoyanovsky, J. S. Greenberger, G. Borisenko, N. A.

Belikova, N. Yanamala, A. K. Samhan Arias, M. A. Tungekar, J. Jiang, Y. Y. Tyurina, J. Ji, J. Klein-Seetharaman, B. R. Pitt, A. A. Shvedova, H. Bayir, Adv. Drug Delivery Rev. 2009, 61, 1375.

[22] A. Dhanasekaran, S. Kotamraju, C. Karunakaran, S. V. Kalivendi, S. Thomas, J. Joseph, B. Kalyanaraman, Free Radical Biol. Med. 2005, 39, 567.

[23] J. Dessolin, M. Schuler, A. Quinart, F. De Giorgi, L. Ghosez, F. Ichas, Eur. J. Pharmacol. 2002, 447, 155.

[24] a) N. Bertrand, J. Wu, X. Xu, N. Kamaly, O. C. Farokhzad, Adv. Drug Delivery Rev. 2014, 66, 2; b) R. A. Petros, J. M. DeSimone, Nat. Rev. Drug Discovery. 2010, 9, 615. [25] a) B. H. Nguyen, V. H. Nguyen, Adv. Nat. Sci.: Nanosci. Nanotechnol. 2016, 7 , 023002; b) V. Georgakilas, M. Otyepka, A. B. Bourlinos, V. Chandra, N. Kim, K. C. Kemp, P. Hobza, R. Zboril, K. S. Kim, Chem. Rev. 2012, 112, 6156.

[26] a) A. Gulzar, P. Yang, F. He, J. Xu, D. Yang, L. Xu, M. O. Jan, Chem. -Biol. Interact. 2017, 262, 69; b) X. Sun, Z. Liu, K. Welsher, J. T. Robinson, A. Goodwin, S. Zaric, H. Dai, Nano Res. 2008, 1, 203. 


\section{WILEY-VCH}

[27] K. Yang, L. Feng, X. Shi, Z. Liu, Chem. Soc. Rev. 2013, 42, 530.

[28] Z. M. Markovic, B. Z. Ristic, K. M. Arsikin, D. G. Klisic, L. M. Harhaji-Trajkovic, B. M. Todorovic-Markovic, D. P. Kepic, T. K. Kravic-Stevovic, S. P. Jovanovic, M. M.

Milenkovic, D. D. Milivojevic, V. Z. Bumbasirevic, M. D. Dramicanin, V. S. Trajkovic, Biomaterials 2012, 33, 7084.

[29] J. Ge, M. Lan, B. Zhou, W. Liu, L. Guo, H. Wang, Q. Jia, G. Niu, X. Huang, H. Zhou, X. Meng, P. Wang, C. S. Lee, W. Zhang, X. Han, Nat. Commun. 2014, 5, 4596.

[30] D. R. Dreyer, S. Park, C. W. Bielawski, R. S. Ruoff, Chem. Soc. Rev. 2010, 39, 228.

[31] a) D. K. Chatterjee, L. S. Fong, Y. Zhang, Adv. Drug Delivery Rev. 2008, 60, 1627; b)

A. Vogel, V. Venugopalan, Chem. Rev. 2003, 103, 577.

[32] a) J. Peng, W. Gao, B. K. Gupta, Z. Liu, R. Romero-Aburto, L. Ge, L. Song, L. B. Alemany, X. Zhan, G. Gao, S. A. Vithayathil, B. A. Kaipparettu, A. A. Marti, T. Hayashi, J.J. Zhu, P. M. Ajayan, Nano Lett. 2012, 12, 844; b) R. Ye, C. Xiang, J. Lin, Z. Peng, K. Huang, Z. Yan, N. P. Cook, E. L. G. Samuel, C.-C. Hwang, G. Ruan, G. Ceriotti, A.-R. O. Raji, A. A. Martí, J. M. Tour, Nat. Commun. 2013, 4, 2943.

[33] a) B. Zhao, J.-J. Yin, P. J. Bilski, C. F. Chignell, J. E. Roberts, Y.-Y. He, Toxicol. Appl. Pharmacol. 2009, 241, 163; b) M. Wojtoniszak, D. Rogińska, B. Machaliński, M. Drozdzik, E. Mijowska, Mater. Res. Bull. 2013, 48, 2636.

[34] L. Xiao, L. Gu, S. B. Howell, M. J. Sailor, ACS Nano 2011, 5, 3651.

[35] R. Vankayala, A. Sagadevan, P. Vijayaraghavan, C. L. Kuo, K. C. Hwang, Angew. Chem., Int. Ed. Engl. 2011, 50, 10640.

[36] a) J. J. M. Lamberts, D. R. Schumacher, D. C. Neckers, J. Am. Chem. Soc. 1984, 106, 5879; b) R. W. Redmond, J. N. Gamlin, Photochem. Photobiol. 1999, 70, 391.

[37] a) Y. Sui, K. Tao, Q. Tian, K. Sun, J. Phys. Chem. C 2012, 116, 1732; b) Q. Tian, K. Tao, W. Li, K. Sun, J. Phys. Chem. C 2011, 115, 22886.

[38] Q. Q. Dou, C. P. Teng, E. Ye, X. J. Loh, Int. J. Nanomed. 2015, 10, 419.

[39] S. T. Smiley, M. Reers, C. Mottola-Hartshorn, M. Lin, A. Chen, T. W. Smith, G. D.

Steele, L. B. Chen, Proc. Natl. Acad. Sci. U. S. A. 1991, 88, 3671.

[40] a) Y. Lu, P. S. Low, Adv. Drug Delivery Rev. 2012, 64, 342; b) B. Bahrami, M. Mohammadnia-Afrouzi, P. Bakhshaei, Y. Yazdani, G. Ghalamfarsa, M. Yousefi, S. Sadreddini, F. Jadidi-Niaragh, M. Hojjat-Farsangi, Tumor Biol. 2015, 36, 5727; c) L. M. Bareford, P. W. Swaan, Adv. Drug Delivery Rev. 2007, 59, 748.

[41] a) M. P. Murphy, R. A. Smith, Annu. Rev. Pharmacol. Toxicol. 2007, 47, 629; b) H. Hillaireau, P. Couvreur, Cell. Mol. Life Sci. 2009, 66, 2873.

[42] a) W. J. Chang, K. G. Rothberg, B. A. Kamen, R. G. Anderson, J. Cell Biol. 1992, 118, 63; b) E. Dauty, J.-S. Remy, G. Zuber, J.-P. Behr, Bioconjugate Chem. 2002, 13, 831.

[43] a) L. Pelkmans, A. Helenius, Traffic 2002, 3, 311; b) G. Bathori, L. Cervenak, I. Karadi, Crit. Rev. Ther. Drug Carrier Syst. 2004, 21, 30.

[44] a) L. Milane, M. Trivedi, A. Singh, M. Talekar, M. Amiji, J. Controlled Release 2015, 207, 40; b) O. M. de Brito, L. Scorrano, The EMBO Journal. 2010, 29, 2715; c) T. Daniele, M. V. Schiaffino, Commun. Integr. Biol. 2014, 7, 29587.

[45] a) Y. Wei, F. Zhou, D. Zhang, Q. Chen, D. Xing, Nanoscale 2016, 8, 3530; b) J. Xu, F. Zeng, H. Wu, C. Hu, S. Wu, Biomacromolecules 2014, 15, 4249.

[46] a) J. Xu, F. Zeng, H. Wu, C. Yu, S. Wu, ACS Appl. Mater. Interfaces 2015, 7, 9287; b) G. Battogtokh, Y. T. Ko, Nanomedicine 2017, 13, 733. 


\section{WILEY-VCH}

Synergistic targeting strategy was established by simultaneously modifying a tumortargeting motif and a mitochondria-targeting agent onto graphene oxide quantum dotsupconversion nanocrystals hybrid nanoparticles. Under the non-interfering impetus of receptor-mediated endocytosis and electrostatic interaction, cellular uptake and mitochondriatargeting efficiency for Hela cells were significantly improved, so that mitochondria damage and in vivo therapeutic effects was enhanced with this synergistic targeting modification.

Keyword: photodynamic therapy, upconversion nanoparticles, graphene oxide quantum dots, synergistic targeting, subcellular location

Yan Liu†, Yawen Xu†, Xiangshuai Geng†, Dexin Chen, Kang Sun, Yingying Huo, Guangdong Zhou, Biqiong Chen*, Ke Tao*

Synergistic targeting and efficient photodynamic therapy based on graphene oxide quantum dot-upconversion nanocrystal hybrid nanoparticles

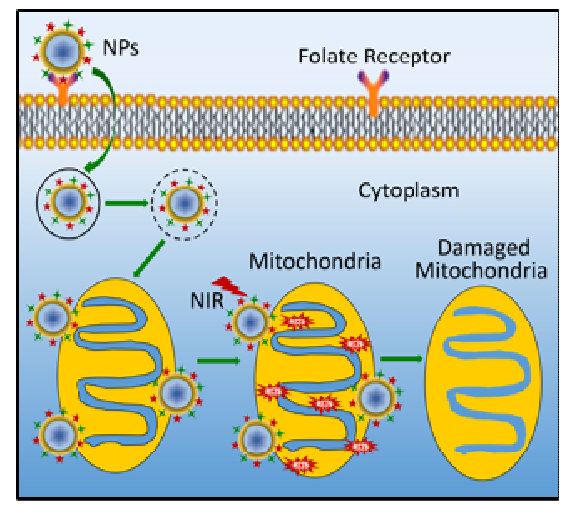

